\title{
Optimal Convergence Rates for Solutions of the Monopolar Non-Newtonian Flows
}

\author{
Zhi-qiang Luo \\ Department of System Science and Applied Mathematics, Kunming University of Science and Technology, Kunming 650083, China \\ Correspondence should be addressed to Zhi-qiang Luo; zq11009@126.com
}

Received 22 May 2014; Accepted 1 July 2014; Published 9 July 2014

Academic Editor: Bo-Qing Dong

Copyright (c) 2014 Zhi-qiang Luo. This is an open access article distributed under the Creative Commons Attribution License, which permits unrestricted use, distribution, and reproduction in any medium, provided the original work is properly cited.

This paper is concerned with the optimal convergence rates for solutions of the monopolar non-Newtonian flows. By using the energy methods, the perturbed weak solution of perturbed system asymptotically converges to the solution of the original system with the optimal rates $(1+t)^{-1 / 4}$.

\section{Introduction}

In this paper we consider the monopolar non-Newtonian flows which is governed by the following system:

$$
\begin{gathered}
\partial_{t} u+(u \cdot \nabla) u-\nabla \cdot \tau^{v}+\nabla \pi=f, \\
\nabla \cdot u=0, \\
u(x, 0)=u_{0} .
\end{gathered}
$$

Here $u=u(x, t)=\left(u_{1}, u_{2}, u_{3}\right)$ and $\pi$ denote the unknown velocity vector and pressure of the fluids, while $u_{0}$ is the given initial velocity and $f$ is the external force. $\tau^{v}=\left(\tau_{i j}^{v}\right)$ is the stress tensor specified in the following form:

$$
\tau_{i j}^{v}=2\left(\mu_{1}+\mu_{2}|e(u)|^{p-2}\right) e_{i j}(u)
$$

for the symmetric deformation velocity tensor $e(u)=\left(e_{i j}(u)\right)$ with

$$
e_{i j}(u)=\frac{1}{2}\left(\frac{\partial u_{i}}{\partial x_{j}}+\frac{\partial u_{j}}{\partial x_{i}}\right),
$$

where $\mu_{1}>0, \mu_{2}>0$ are viscous coefficients.

The system (1) was proposed for the first time by Ladyzhenskaya [1] which describes viscous non-Newtonian flows such as the molten plastics, dyes, adhesives, paints, and greases. The constitutive of those fluids is nonlinear and does not the Stokes laws. It should be mentioned that the fluid considered here is monopolar since only first order derivative of the velocity field is involved. When $\mu_{2}=0$, the system becomes the classic Navier-Stokes equations [2].

Due to the importance in both mathematics and physics, the well-posedness and large time behavior of incompressible fluids have attracted more and more attention [3-6]. We refer to some classic results on the Navier-Stokes equations [3]. For the viscous non-Newtonian flows (1), there is also a large literature on the well-posedness and asymptotic behaviors [7-10]. In particular, Pokory [11] and Bae [12] recently studied the existence and uniqueness for the Cauchy problem of the system (1). When $f=0$ by developing the Fourier splitting method, Dong and Li [13] explored the optimal algebraic decay rate in $\mathbb{R}^{3}$ as

$$
\|u(t)\|_{L^{2}} \leq C(1+t)^{-1 / 2}, \quad \forall t>0 .
$$

It should be mentioned that the time decay properties for weak solutions of the non-Newtonian fluids (1) essentially show that the trivial solution $u=0$ is stable. It is desirable to consider the stability of the nontrivial solutions of the nonNewtonian fluids (1) with the nonzero and nondecay external force. To do so, we consider the perturbed non-Newtonian fluids

$$
\begin{gathered}
\partial_{t} v+(v \cdot \nabla) v-\nabla \cdot \tau^{v}+\nabla \pi=f \\
\nabla \cdot v=0, \\
v(x, 0)=u_{0}+w_{0},
\end{gathered}
$$


where $w_{0}$ is the initial perturbation. It is easy to check that if the external force satisfies $f \in L^{2}\left(0, T ; L^{2}\left(\mathbb{R}^{3}\right)\right)$, the nonNewtonian fluids (1) has nontrivial stationary solution and $u=0$ is not a solution of (1) any more. In the two-dimensional case, Dong and Chen [14] have considered the stability issue as

$$
\|v(t)-u(t)\| \longrightarrow 0, \quad \text { as } t \longrightarrow \infty,
$$

for weak solutions of the non-Newtonian fluids (1) under any perturbation $w_{0}$. It should be mentioned that Dong and Chen [14] cannot study the optimal convergence rates. One may also refer to some interesting stability results on the classic Navier-Stokes equations. When $f=0, w_{0} \in L^{1}\left(\mathbb{R}^{3}\right) \cap L^{r}\left(\mathbb{R}^{3}\right)$ $(r>3)$ with $\left\|w_{0}\right\|_{L^{r}}$ sufficiently small; da Veiga and Secchi [15] proved that there is a unique global solution $v(x, t)$ of perturbed Navier-Stokes equations satisfying

$$
\|u(t)-v(t)\|_{L^{r}\left(\mathbb{R}^{3}\right)} \leq C(1+t)^{-3 / 4} \quad \forall t>0
$$

for the weak solution $u(x, t)$ of the original Navier-Stokes equations in the subcritical class

$$
u(x, t) \in L^{\infty}\left(0, \infty ; L^{r+2}\left(\mathbb{R}^{3}\right)\right) .
$$

When $f=0$, Ponce et al. [16] further considered the weak solution $u(x, t)$ of the original Navier-Stokes equations under the critical space

$$
\nabla u \in L^{4}\left(0, \infty ; L^{2}\left(\mathbb{R}^{3}\right)\right)
$$

and obtained that if

$$
\left\|w_{0}\right\|_{H^{1}} \leq \delta
$$

for the sufficient small constant $\delta>0$, then there is a unique solution $v(t)$ of perturbed Navier-Stokes equations satisfying

$$
\sup _{t>0}\|u(t)-v(t)\|_{H^{1}\left(\mathbb{R}^{3}\right)} \leq M(\delta),
$$

where $M(\delta)$ is a constant satisfying $\lim _{\delta \rightarrow 0} M(\delta)=0$. One may also refer to some related results on the Newtonian and viscous non-Newtonian flows [17, 18].

In this paper, we will consider the stability for weak solution of three-dimensional non-Newtonian fluids (1) and will derive the optimal convergence rates. More precisely, we will show that every perturbed solution $v$ of the nonNewtonian fluids (2) converges asymptotically to $u$ of the non-Newtonian fluids (1) as

$$
\|v(t)-u(t)\|_{L^{2}}=O\left((1+t)^{-1 / 4}\right), \quad t \rightarrow \infty .
$$

\section{Preliminaries and Main Results}

Throughout this paper, $C$ stands for a generic positive constant which may vary from line to line. $L^{p}\left(\mathbb{R}^{3}\right)$ with $1 \leq$ $p \leq \infty$ denotes the usual Lebesgue space [19] of all $L^{p}$ integral functions associated with the norm

$$
\|f\|_{L^{p}}= \begin{cases}\left(\int_{\mathbb{R}^{3}}|f(x)|^{p} d x\right)^{1 / p}, & 1 \leq p<\infty, \\ \underset{x \in \mathbb{R}^{3}}{\operatorname{ess} \sup ^{3}|f(x)|,} & p=\infty .\end{cases}
$$

We denote the Fourier transformation $\widehat{f}$ or $F[f]$ as

$$
F f(x)=\widehat{f}(x)=\frac{1}{(2 \pi)^{3 / 2}} \int_{\mathbb{R}^{3}} f(y) e^{-i x \cdot y} d y .
$$

We recall the following Gronwall inequality which will be used in the following argument.

Lemma 1 (Gronwall inequality [2]). Let $f(t), g(t), h(t)$ be nonnegative continuous functions and satisfy the following inequality:

$$
g(t) \leq f(t)+\int_{0}^{t} g(s) h(s) d s, \quad \forall t>0,
$$

where $f^{\prime}(t) \geq 0$. Then

$$
g(t) \leq f(t) \exp \left(\int_{0}^{t} h(s) d s\right), \quad \forall t>0 .
$$

We now give the definition for weak solutions of threedimensional non-Newtonian fluids (1) (see [11]).

Definition 2. Suppose $p \geq 3, u_{0} \in L^{2}\left(\mathbb{R}^{3}\right), f \in L^{2}\left(0, T ; L^{2}\right.$ $\left(\mathbb{R}^{3}\right)$ ), and $u(x, t)$ is called a weak solution of the nonNewtonian fluids (1) if the following conditions hold true.

(i) $u(x, t) \in L^{\infty}\left(0, T ; L^{2}\left(\mathbb{R}^{3}\right)\right)$ and $\nabla u \in L^{p}\left(0, T ; L^{p}\left(\mathbb{R}^{3}\right)\right)$ $\cap L^{2}\left(0, T ; L^{2}\left(\mathbb{R}^{3}\right)\right)$ for all $T>0$.

(ii) $u$ is weakly continuous from $[0, \infty)$ to $L^{2}\left(\mathbb{R}^{3}\right)$.

(iii) $u$ satisfies (1) in the weak sense; that is, for all $\varphi \in$ $C^{1}\left([s, t] ; \dot{H}^{1}\right)$

$$
\begin{gathered}
\int_{\mathbb{R}^{3}} u(t) \varphi(t) d x+\int_{s}^{t} \int_{\mathbb{R}^{3}}\left(\tau^{v} \cdot \nabla \varphi+(u \cdot \nabla) u \varphi\right) d x d \tau \\
=\int_{s}^{t} \int_{\mathbb{R}^{3}} u \partial_{\tau} \varphi d x d \tau+\int_{\mathbb{R}^{3}} u(s) \varphi(s) d x \\
\quad+\int_{s}^{t} \int_{\mathbb{R}^{3}} f \varphi d x d \tau .
\end{gathered}
$$

(iv) $u$ satisfies energy inequality; for all $t \geq 0$

$$
\begin{gathered}
\int_{\mathbb{R}^{3}}|u(t)|^{2} d x+2 \mu_{1} \int_{0}^{t} \int_{\mathbb{R}^{3}}|\nabla u(\tau)|^{2} d x d \tau \\
+2 \mu_{2} \int_{0}^{t} \int_{\mathbb{R}^{3}}|\nabla u(\tau)|^{p} d x d \tau \\
\leq \int_{\mathbb{R}^{3}}\left|u_{0}\right|^{2} d x+2 \int_{s}^{t} \int_{\mathbb{R}^{3}} f u d x d \tau .
\end{gathered}
$$

The following existence and regularity of weak solutions of the incompressible non-Newtonian fluids (1) are due to the work of Pokory [11].

Lemma 3 (existence and regularity of weak solutions). Suppose $p \geq 3, u_{0} \in L^{2}\left(\mathbb{R}^{3}\right) \cap \dot{H}^{1}\left(\mathbb{R}^{3}\right)$, and $f \in L^{2}\left(0, T ; L^{2}\left(\mathbb{R}^{3}\right)\right)$. 
Then there exists a weak solution of the incompressible nonNewtonian fluids (1). Moreover, the solution is regular; that is,

$$
\begin{array}{r}
\nabla u \in L^{\infty}\left(0, T ; L^{2}\left(\mathbb{R}^{3}\right)\right), \\
\Delta u \in L^{2}\left(0, T ; L^{2}\left(\mathbb{R}^{3}\right)\right), \\
\forall T>0 .
\end{array}
$$

Our results now read.

Theorem 4. Suppose $p \geq 3$ and $u(x, t)$ is a weak solution of the incompressible non-Newtonian fluids (1) with the finite energy initial data $u_{0} \in L^{2}\left(\mathbb{R}^{3}\right) \cap \dot{H}^{1}\left(\mathbb{R}^{3}\right)$ and the nondecay external force $f \in L^{2}\left(0, T ; L^{2}\left(\mathbb{R}^{3}\right)\right)$. Then for any initial perturbation $w_{0} \in L^{2}\left(\mathbb{R}^{3}\right) \cap L^{1}\left(\mathbb{R}^{3}\right)$, the perturbed non-Newtonian fluids (2) has a weak solution $v(x, t)$ which asymptotically converges $u(x, t)$ with the optimal convergence rate:

$$
\|v(t)-u(t)\|_{L^{2}}=O\left((1+t)^{-1 / 4}\right), \quad t \longrightarrow \infty .
$$

Remark 5. Compared with some stability results of the classic Navier-Stokes equations such as da Veiga and Secchi [15] and Ponce et al. [16], we do not have any small assumptions on the initial perturbation. Moreover, our results also improve the previous results obtained by Dong and Chen [17] since we not only consider this problem in three-dimensional case instead of the two-dimensional case, but also derive the optimal convergence rates $(20)$.

Remark 6. The proof of Theorem 4 is mainly based on Fourier splitting technique. The nonlinear system is essentially also a dissipative system which may allow us to divide the frequency domain into two time-dependent subdomains; the subdomain yields a first-order differential inequality for the spatial $L^{2}$ norm of the Fourier transform of the weak solutions of incompressible non-Newtonian fluids (1). Compared with the derivation of Dong and Chen [17], the estimates of the nonlinear term can be dealt with in a satisfied form, for the three-dimensional case; however, the bounds of the nonlinear term become difficult. In order to overcome, we make full use of the regular properties of weak solutions and energy methods.

\section{Proof of Theorem 4}

According to the condition in Theorem 4 and the existence results of non-Newtonian fluids (1) obtained in [11], the existence of weak solutions of the perturbation of the perturbed incompressible non-Newtonian fluids (5) can be obtained by the parallel methods in [11]. Therefore, what remains is to prove the optimal convergence rates (20).

Let $\left(u, \pi_{1}\right)$ and $\left(v, \pi_{2}\right)$ be weak solutions of the original non-Newtonian fluids (1) and the perturbed non-Newtonian fluids (5), respectively. We denote by $w=v-u$ and $\pi=\pi_{2}-$ $\pi_{1}$ the difference between the two weak solutions of (1) and (2); then $w$ satisfies the following system in the weak sense:

$$
\begin{aligned}
& w_{t}-\mu_{1} \Delta w+(v \cdot \nabla) w+(w \cdot \nabla) u \\
& \quad-\mu_{2}\left(\operatorname{div}\left(|\nabla v|^{p-2} \nabla v\right)-\operatorname{div}\left(|\nabla u|^{p-2} \nabla u\right)\right) \\
& \quad+\nabla \pi=0
\end{aligned}
$$

$$
\begin{gathered}
\nabla \cdot w=0, \\
u(x, 0)=w_{0} .
\end{gathered}
$$

In order to investigate the optimal convergence rates, we now need some a priori estimates of the nonlinear system (21).

Lemma 7. Under the same condition in Theorem 4, then the difference $w=v-u$ satisfies the following inequality:

$$
\widehat{w}(\xi, t) \leq\left|\widehat{w}_{0}(\xi) e^{-\mu_{1}|\xi|^{2} t}\right|+c|\xi|+c|\xi| t .
$$

Proof of Lemma 7. We first formally derive inequality (22). The rigorous derivation is obtained by considering the approximated solutions of the following approximate system:

$$
\begin{aligned}
& \partial_{t} w_{k}-\mu_{1} \Delta w+\left(v_{k} \cdot \nabla\right) \widetilde{w}_{k}+\left(\widetilde{w}_{k} \cdot \nabla\right) u_{k} \\
& +\nabla \pi=0 \\
& \nabla \cdot w_{k}=0, \\
& w_{k}(x, 0)=w_{0} \text {. }
\end{aligned}
$$$$
-\mu_{2}\left(\operatorname{div}\left(\left|\nabla \widetilde{v}_{k}\right|^{p-2} \nabla v_{k}\right)-\operatorname{div}\left(\left|\nabla \widetilde{u}_{k}\right|^{p-2} \nabla u_{k}\right)\right)
$$

Here the retarded modification $\tilde{f}_{k}$ of $f_{k}$ is defined by

$$
\begin{array}{r}
\tilde{f}_{k}(x, t)=\delta^{-4} \iint \psi\left(\frac{y}{\delta}, \frac{s}{\delta}\right) \bar{f}_{k}(x-y, t-s) d y d s, \\
k=\frac{T}{\delta},
\end{array}
$$

where $\psi \in C_{0}^{\infty}\left(\mathbb{R}^{3} \times(0, \infty)\right)$ is a positive modifier. $\bar{f}_{k}$ is the zero extension of the function $u_{k}$ which is originally defined for $t \geq 0$. For the similar estimates (22) of the approximate solutions $w_{k}$, we only need to take the limit as $k \rightarrow \infty$. Therefore we now deal with this settle in the following argument only for weak solution $w$ directly.

Taking Fourier transformation of (23), it follows that by solving an ordinary equation

$$
\begin{aligned}
\widehat{w} & =\widehat{w}_{0}(\xi) e^{-\mu_{1}|\xi|^{2} t}+\int_{0}^{t} e^{-\mu_{1}|\xi|^{2}(t-s)} \mathcal{N}(\xi, s) d s \\
& \leq \widehat{e^{-\mu_{1} \Delta t} w_{0}}+\int_{0}^{t}|\mathcal{N}(\xi, s)| d s,
\end{aligned}
$$


where

$$
\begin{aligned}
N(\xi, t)= & -F[(v \cdot \nabla) w+(w \cdot \nabla) u] \\
& +F\left[\mu_{2}\left(\operatorname{div}\left(|\nabla v|^{p-2} \nabla v\right)-\operatorname{div}\left(|\nabla u|^{p-2} \nabla u\right)\right)\right] \\
& -F[\nabla p] \\
= & : I+J+K .
\end{aligned}
$$

We now compute $I, J, K$ one by one. For $I$, applying the divergence free properties of the velocity fields and Hölder inequality, it follows that

$$
\begin{aligned}
I & \leq\left|\frac{1}{(2 \pi)^{3 / 2}} \int_{\mathbb{R}^{3}} e^{-i x \cdot y}\{(v \cdot \nabla) w+(w \cdot \nabla) u\} d y\right| \\
& \leq \sum_{i, j}\left|\frac{1}{(2 \pi)^{3 / 2}} \int_{\mathbb{R}^{3}} e^{-i x \cdot y}\left\{\partial_{i}\left(v_{i} w_{j}\right)+\partial_{i}\left(w_{i} u_{j}\right)\right\} d y\right| \\
& \leq C \sum_{i, j}|\xi| \int_{\mathbb{R}^{3}}\left|v_{i} w_{j}+w_{i} u_{j}\right| d y \\
& \leq C|\xi|\left(\|v\|_{L^{2}}+\|u\|_{L^{2}}\right)\|w\|_{L^{2}} \\
& \leq C|\xi|
\end{aligned}
$$

where we have used the bounds of $u, v, w$; that is,

$$
\text { ess } \sup _{t}\left(\|v\|_{L^{2}}+\|u\|_{L^{2}}\right) \leq C, \quad \text { ess } \sup _{t}\|w\|_{L^{2}} \leq C .
$$

For $J$, one shows that

$$
\begin{aligned}
J & \leq \mid \frac{1}{(2 \pi)^{3 / 2}} \int_{\mathbb{R}^{3}} e^{-i x \cdot y}\left\{\mu _ { 2 } \left(\operatorname{div}\left(|\nabla v|^{p^{-2}} \nabla v\right)\right.\right. \\
& \left.\left.-\operatorname{div}\left(|\nabla u|^{p-2} \nabla u\right)\right)\right\} d y \mid \\
& \leq\left. C|\xi| \int_{\mathbb{R}^{3}}|| \nabla u\right|^{p-2} \nabla u+|\nabla v|^{p-2} \nabla v \mid d y \\
& \leq C|\xi|\left(\|\nabla v\|_{L^{p-1}}^{p-1}+\|\nabla u\|_{L^{p-1}}^{p-1}\right) .
\end{aligned}
$$

In order to estimate $K$, we act the divergence operator to both sides of (21); it follows that

$$
\begin{aligned}
\Delta \pi & =\sum_{i, j} \frac{\partial^{2}}{\partial x_{i} \partial x_{j}}\left(-v_{i} w_{j}-u_{i} w_{j}+|\nabla u|^{p-2} \partial_{i} u_{j}-|\nabla v|^{p-2} \partial_{i} v_{j}\right), \\
|\xi|^{2} F[\pi] & \\
& =\sum_{i, j} \xi_{i} \xi_{j} F\left[-v_{i} w_{j}-u_{i} w_{j}+|\nabla u|^{p-2} \partial_{i} u_{j}-|\nabla v|^{p-2} \partial_{i} v_{j}\right] .
\end{aligned}
$$

Now together with the estimates of $I$ and $J, K$ can be estimated as

$$
K \leq C|\xi|+C|\xi|\left(\|\nabla v\|_{L^{p-1}}^{p-1}+\|\nabla u\|_{L^{p-1}}^{p-1}\right) .
$$

Hence plugging the estimates (27)-(31) of $I, J, K$ into (26) and then (25), we may obtain that

$$
|\widehat{w}| \leq \widehat{e^{-\mu_{1} \Delta t} w_{0}}+C|\xi| t+C|\xi| \int_{0}^{t}\left(\|\nabla v\|_{L^{p-1}}^{p-1}+\|\nabla u\|_{L^{p-1}}^{p-1}\right) d s .
$$

Thanks to

$$
\begin{aligned}
& \left(\int_{0}^{\infty}\|\nabla u\|_{p-1}^{p-1} d s\right)^{1 /(p-1)} \\
& \quad \leq\left(\int_{0}^{\infty}\|\nabla u\|_{2}^{\theta(p-1)}\|\nabla u\|_{p}^{(1-\theta)(p-1)} d s\right)^{1 /(p-1)} \\
& \quad \leq\left(\int_{0}^{\infty}\|\nabla u\|_{2}^{2} d s\right)^{\theta / 2}\left(\int_{0}^{\infty}\|\nabla u\|_{p}^{p} d s\right)^{(1-\theta) / p} \\
& \quad \leq C
\end{aligned}
$$

for

$$
\frac{1}{p-1}=\frac{\theta}{2}+\frac{1-\theta}{p}
$$

and the following facts due to the energy inequality

$$
\nabla u, \nabla v \in L^{2}\left(0, \infty ; L^{2}\right) \cap L^{p}\left(0, \infty ; L^{p}\right) \quad \text { for } p \geq 3,
$$

thus (32) is written as

$$
|\widehat{w}| \leq\left|e^{\widehat{\mu_{1} \Delta t} w_{0}}\right|+C|\xi| t+C|\xi|
$$

which completes the proof of this lemma.

Now we are in positive to prove (20). Taking the $L^{2}$ inner product of (21) with $w$, it follows that

$$
\frac{1}{2} \frac{d}{d t} \int_{\mathbb{R}^{3}}|w|^{2} d x+\mu_{1} \int_{\mathbb{R}^{3}}|\nabla w|^{2} d x \leq \int_{\mathbb{R}^{3}}(w \cdot \nabla) w \cdot u d x,
$$

where we have used the following fact:

$$
\begin{gathered}
\int_{\mathbb{R}^{3}} \mu_{2}\left(|\nabla v|^{p-2} \nabla v-|\nabla u|^{p-2} \nabla u\right) \cdot \nabla w d x \\
\geq \mu_{2}\left\{\|\nabla v\|_{L^{p}}^{p}-\|\nabla u\|_{L^{p}}^{p}+\|\nabla v\|_{L^{p}}^{p-1}\|\nabla u\|_{L^{p}}\right. \\
\left.-\|\nabla u\|_{L^{p}}^{p-1}\|\nabla v\|_{L^{p}}\right\} \\
\geq\left(\|\nabla v\|_{L^{p}}^{p-1}-\|\nabla u\|_{L^{p}}^{p-1}\right)\left(\|\nabla v\|_{L^{p}}-\|\nabla u\|_{L^{p}}\right) \geq 0 .
\end{gathered}
$$

Applying Hölder inequality, Gagliar-Nirenberg inequality, and Young inequality gives that

$$
\begin{aligned}
\left|\int_{\mathbb{R}^{3}}(w \cdot \nabla) w \cdot u d x\right| & \leq C\|w\|_{L^{3}}\|\nabla w\|_{L^{2}}\|u\|_{L^{6}} \\
& \leq C\|\nabla w\|_{L^{2}}^{3 / 2}\|w\|_{L^{2}}^{1 / 2}\|\nabla u\|_{L^{2}} \\
& \leq \frac{\mu_{1}}{2}\|\nabla w\|_{L^{2}}^{2}+C\left(\mu_{1}\right)\|w\|_{L^{2}}^{2}\|\nabla u\|_{L^{2}}^{4}
\end{aligned}
$$


from which we rewrite (37) as

$$
\frac{d}{d t}\|w(t)\|_{L^{2}}^{2}+\mu_{1}\|\nabla w(t)\|_{L^{2}}^{2} \leq C\left(\mu_{1}\right)\|w\|_{L^{2}}^{2}\|\nabla u\|_{L^{2}}^{4} .
$$

Taking Parseval inequality into consideration

$$
\begin{aligned}
& \frac{d}{d t} \int_{\mathbb{R}^{3}}|\widehat{w}(t)|^{2} d \xi+\mu_{1} \int_{\mathbb{R}^{3}}|\xi|^{2}|\widehat{w}(t)|^{2} d \xi \\
& \quad \leq C\left(\mu_{1}\right)\|w\|_{L^{2}}^{2}\|\nabla u\|_{L^{2}}^{4} .
\end{aligned}
$$

And then multiplying both sides by $(1+t)^{4}$

$$
\begin{aligned}
& \frac{d}{d t}\left((1+t)^{3} \int_{\mathbb{R}^{3}}|\widehat{w}(t)|^{2} d \xi\right)+\mu_{1}(1+t)^{4} \int_{\mathbb{R}^{3}}|\xi|^{2}|\widehat{w}(t)|^{2} d \xi \\
& \quad \leq 4(1+t)^{3} \int_{\mathbb{R}^{3}}|\widehat{w}(t)|^{2} d \xi+C(1+t)^{4}\|\widehat{w}\|_{L^{2}}^{2}\|\nabla u\|_{L^{2}}^{4} .
\end{aligned}
$$

Let

$$
S(t)=\left\{\xi \in \mathbb{R}^{3}:|\xi|^{2} \leq \frac{4}{\mu_{1}(1+t)}\right\},
$$

and then we divide the domain $\mathbb{R}^{3}$ of the second integral in (37) into $S(t)$ and $S(t)^{c}$; that is to say,

$$
\begin{aligned}
& \frac{d}{d t}\left((1+t)^{4} \int_{\mathbb{R}^{3}}|\widehat{w}(t)|^{2} d \xi\right) \\
& \quad \leq C(1+t)^{3} \int_{S(t)}|\widehat{w}(t)|^{2} d \xi+C(1+t)^{4}\|\widehat{w}\|_{L^{2}}^{2}\|\nabla u\|_{L^{2}}^{4} .
\end{aligned}
$$

Integrating in time from 0 to $t$ gives

$$
\begin{aligned}
(1+t)^{4} \int_{\mathbb{R}^{3}}|\widehat{w}(t)|^{2} d \xi & \\
\leq & C\left\|\widehat{w}_{0}\right\|_{L^{2}}^{2}+C \int_{0}^{t}(1+s)^{3} \int_{S(s)}|\widehat{w}(s)|^{2} d \xi d s \\
& +\int_{0}^{t}(1+t)^{4}\|\widehat{w}\|_{L^{2}}^{2}\|\nabla u\|_{L^{2}}^{4} d s .
\end{aligned}
$$

By employing Lemma 7, we have after the direct computation

$$
\begin{aligned}
& \int_{0}^{t}(1+s)^{3} \int_{S(s)}|\widehat{w}(s)|^{2} d \xi d s \\
& \leq C \int_{0}^{t}(1+s)^{3} \int_{S(s)}\left|\widehat{e^{-\mu_{1} \Delta t} w_{0}}+\right| \xi|s+| \xi||^{2} d \xi d s \\
& \leq C \int_{0}^{t}(1+s)^{3}\left\|\widehat{e}^{-\mu_{1} \Delta t} w_{0}\right\|_{L^{2}}^{2} d s+C(1+t)^{7 / 2} \\
& +C(1+t)^{3 / 2} \\
& \leq C(1+t)^{7 / 2} \text {, }
\end{aligned}
$$

where we have used the following $L^{p}-L^{q}$ estimates of heat equations:

$$
\left\|e^{-\mu_{1} \Delta t} w_{0}\right\|_{L^{q}\left(\mathbb{R}^{3}\right)} \leq C t^{-(3 / 2)(1 / r-1 / q)}\left\|w_{0}\right\|_{L^{r}\left(\mathbb{R}^{3}\right)}, \quad t>0
$$

with $1 \leq r \leq q \leq \infty$.
Hence we insert (46) into (45) and take Gronwall inequality into consideration:

$$
(1+t)^{4}\|w(t)\|^{2} \leq\left\{C(1+t)^{7 / 2}\right\} \exp \left(C \int_{0}^{\infty}\|\nabla u(s)\|_{L^{2}}^{4} d s\right) .
$$

Since

$$
\nabla u \in L^{2}\left(0, \infty ; L^{2}\left(\mathbb{R}^{3}\right)\right) \cap L^{\infty}\left(0, \infty ; L^{2}\left(\mathbb{R}^{3}\right)\right),
$$

then by the interpolation inequality we have

$$
\nabla u \in L^{4}\left(0, \infty ; L^{2}\left(\mathbb{R}^{3}\right)\right)
$$

Hence (48) implies

$$
\|v(t)-u(t)\|_{L^{2}}=O\left((1+t)^{-1 / 4}\right), \quad t \longrightarrow \infty
$$

which completes the proof of Theorem 4.

\section{Conflict of Interests}

The author declares that there is no conflict of interests regarding the publication of this paper.

\section{Acknowledgment}

This work is partially supported by the Funds of Personnel Training of Kunming University of Science and Technology (no. KKSY201207019).

\section{References}

[1] O. Ladyzhenskaya, "New equations for the description of the viscoue incompressible fluids and solvability in the large of the boundary value problems for them," in Boundary Value Problem of Mathematical Physics V, American Mathematical Society, Providence, RI, USA, 1970.

[2] R. Temam, Navier-Stokes Equations, North-Holland, Amsterdam, The Netherlands, 1977.

[3] L. Caffarelli, R. Kohn, and L. Nirenberg, "Partial regularity of suitable weak solutions of the Navier-Stokes equations," Communications on Pure and Applied Mathematics, vol. 35, no. 6, pp. 771-831, 1982.

[4] F. Lin, "A new proof of the Caffarelli-Kohn-Nirenberg theorem," Communications on Pure and Applied Mathematics, vol. 51, no. 3, pp. 241-257, 1998.

[5] J. Málek, J. Nečas, M. Rokyta, and M. Růžička, Weak and Measure-v alued Solutions to Evolutionary PDEs, ChapmanHall, New York, NY, USA, 1996.

[6] B. Dong and Z. Zhang, "On the weak-strong uniqueness of Koch-Tataru's solution for the Navier-Stokes equations," Journal of Differential Equations, vol. 256, no. 7, pp. 2406-2422, 2014.

[7] B. Dong and Z. Chen, "Asymptotic profiles of solutions to the $2 \mathrm{D}$ viscous incompressible micropolar fluid flows," Discrete and Continuous Dynamical Systems A, vol. 23, no. 3, pp. 765-784, 2009.

[8] B. Dong and Z. Chen, "Regularity criteria of weak solutions to the three-dimensional micropolar flows," Journal of Mathematical Physics, vol. 50, no. 10, Article ID 103525, 13 pages, 2009. 
[9] B. Dong and J. Song, "Global regularity and asymptotic behavior of modified Navier-Stokes equations with fractional dissipation," Discrete and Continuous Dynamical System, vol. 32, no. 1, pp. 57-79, 2012.

[10] Y. Qin, X. Liu, and X. Yang, "Global existence and exponential stability of solutions to the one-dimensional full nonNewtonian fluids," Nonlinear Analysis: Real World Applications, vol. 13, no. 2, pp. 607-633, 2012.

[11] M. Pokory, "Cauchy problem for the non-Newtonian viscous incompressible fluid," Applications of Mathematics, vol. 41, no. 3, pp. 169-201, 1996.

[12] H. Bae, "Existence, regularity, and decay rate of solutions of non-Newtonian flow," Journal of Mathematical Analysis and Applications, vol. 231, no. 2, pp. 467-491, 1999.

[13] B. Dong and Y. Li, "Large time behavior to the system of incompressible non-Newtonian fluids in R2," Journal of Mathematical Analysis and Applications, vol. 298, no. 2, pp. 667-676, 2004.

[14] B. Dong and Z. Chen, "Asymptotic stability of non-Newtonian flows with large perturbation in $\mathrm{R}^{2}$," Applied Mathematics and Computation, vol. 173, no. 1, pp. 243-250, 2006.

[15] H. B. da Veiga and P. Secchi, " $\mathrm{L}^{\mathrm{P}}$-stability for the strong solutions of the Navier-Stokes equations in the whole space," Archive for Rational Mechanics and Analysis, vol. 98, no. 1, pp. 65-69, 1987.

[16] G. Ponce, R. Racke, T. C. Sideris, and E. S. Titi, "Global stability of large solutions to the 3D Navier-Stokes equations," Communications in Mathematical Physics, vol. 159, no. 2, pp. 329-341, 1994.

[17] B. Dong and Z. Chen, "Asymptotic stability of the critical and super-critical dissipative quasi-geostrophic equation," Nonlinearity, vol. 19, no. 12, pp. 2919-2928, 2006.

[18] Y. Zhou, "Asymptotic stability for the 3D Navier-Stokes equations," Communications in Partial Differential Equations, vol. 30, no. 1-3, pp. 323-333, 2005.

[19] H. Bahouri, J.-Y. Chemin, and R. Danchin, Fourier Analysis and Nonlinear Partial Differential Equations, vol. 343 of Fundamental Principles of Mathematical Sciences, Springer, Berlin, Germany, 2011. 


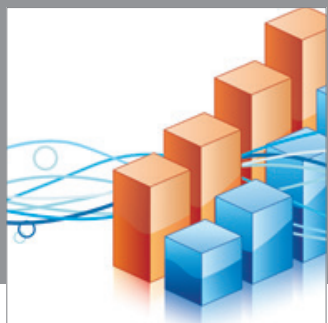

Advances in

Operations Research

mansans

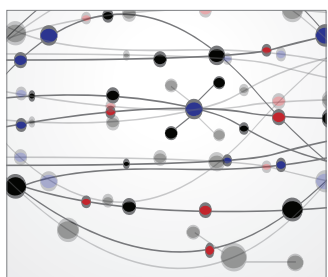

The Scientific World Journal
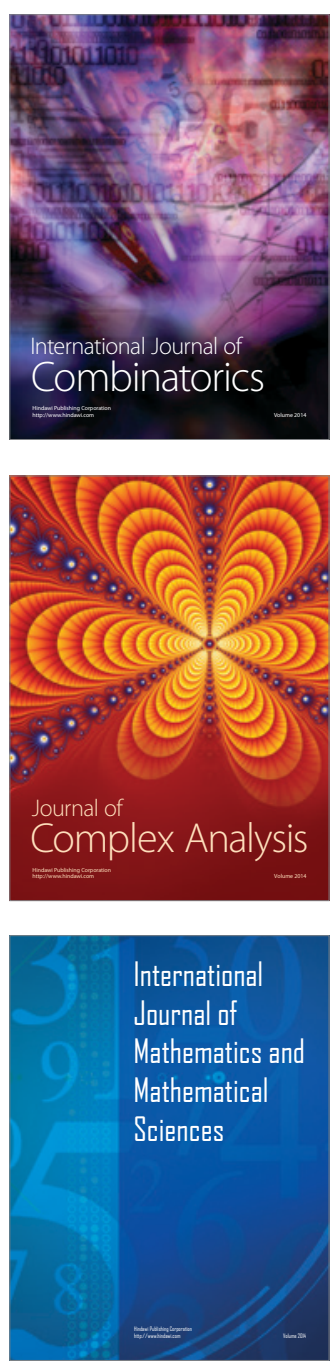
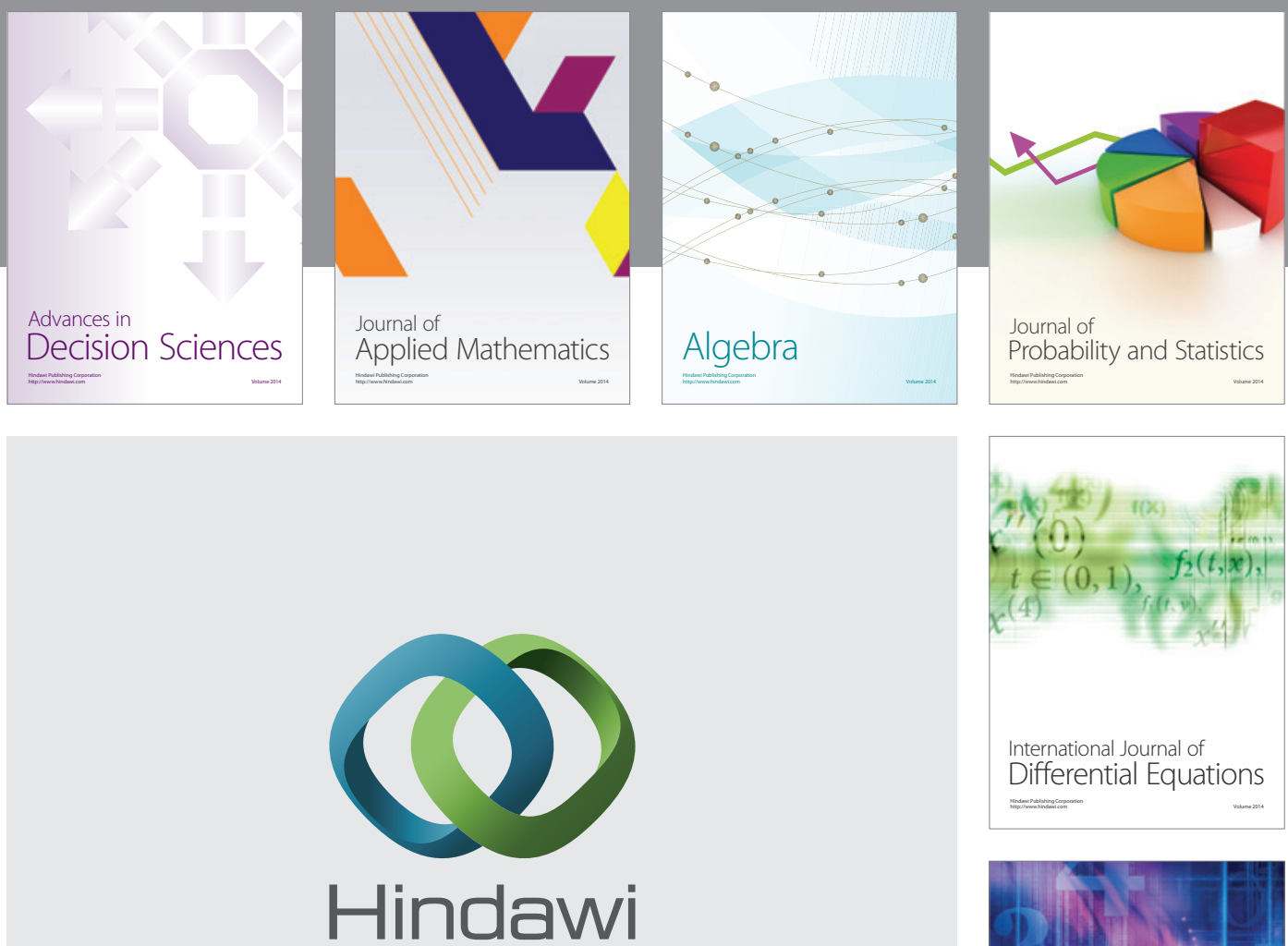

Submit your manuscripts at http://www.hindawi.com
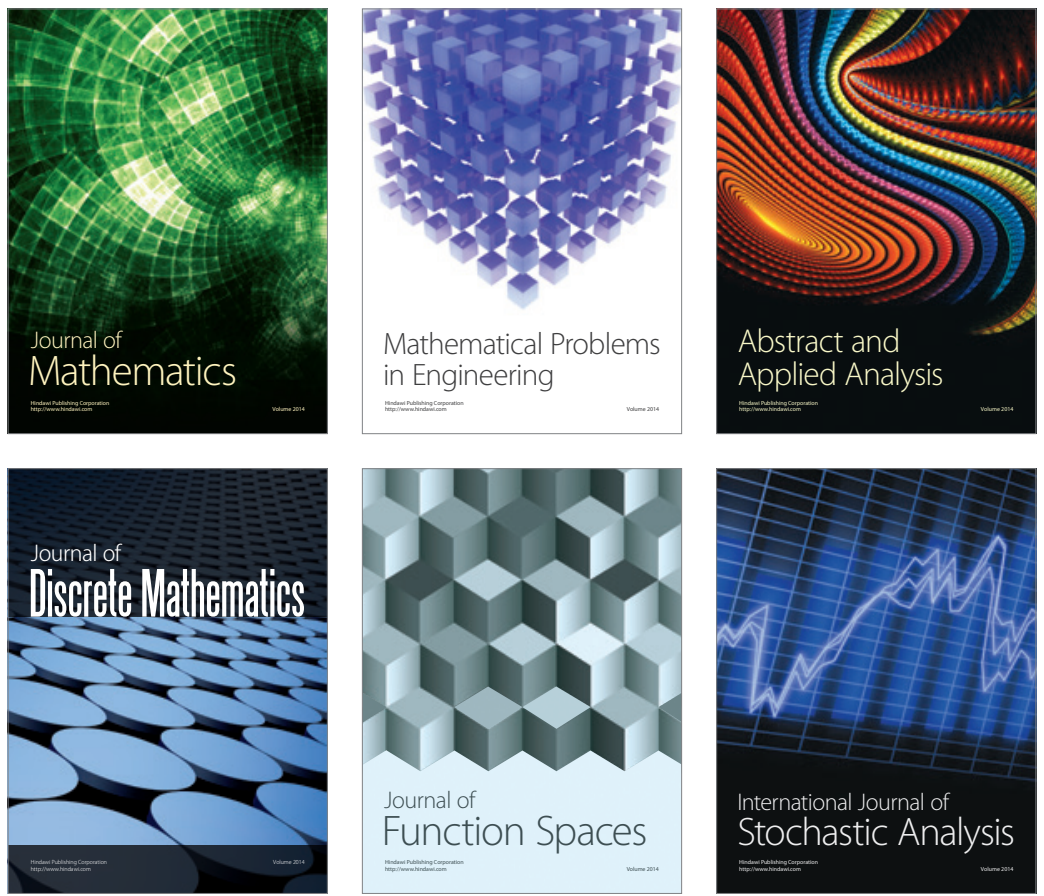

Journal of

Function Spaces

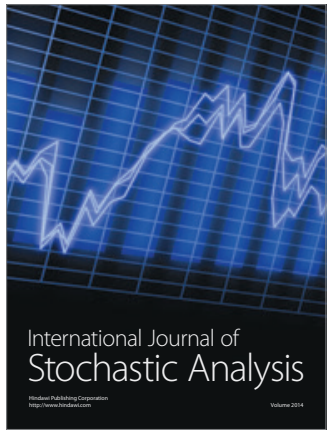

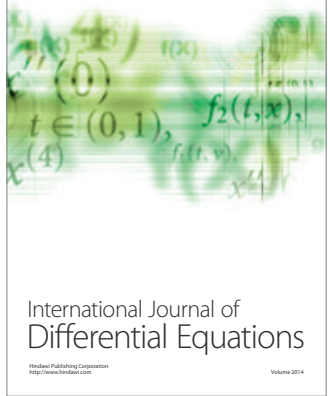
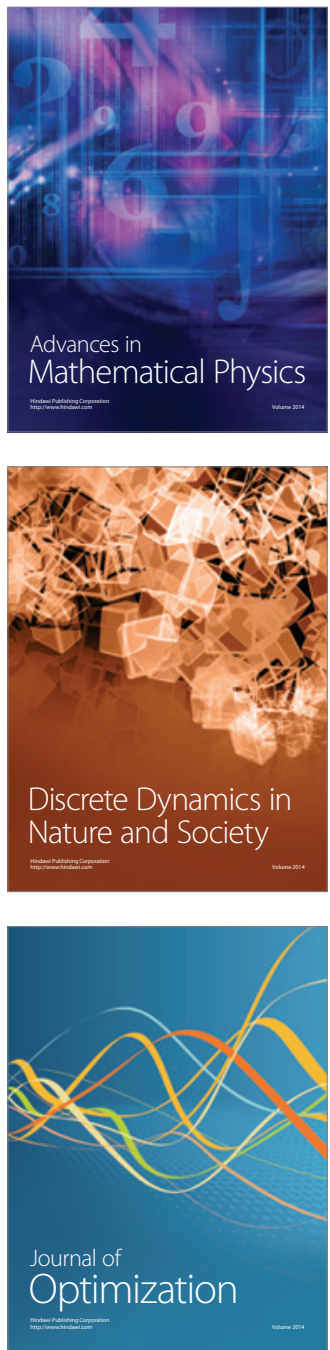